\title{
Cross Cultural Exploration of the Perceived Health Competence Scale
}

\author{
Mary J. Polchert \\ School of Nursing and Health Professions, Marian University, Fond du Lac, WI, USA \\ Email: mjpolchert12@marianuniversity.edu
}

Received 12 June 2015; accepted 12 July 2015; published 15 July 2015

Copyright (C) 2015 by author and Scientific Research Publishing Inc.

This work is licensed under the Creative Commons Attribution International License (CC BY).

http://creativecommons.org/licenses/by/4.0/

(c) (i) Open Access

\section{Abstract}

The eight-item Perceived Health Competence Scale (PHCS) is a measure of self-efficacy in general health management that is used to predict health outcomes and behaviors. The PHCS has been shown to be a reliable and valid instrument but has been used in primarily white European Americans. The PHCS is positively correlated to health status and coping abilities. Younger, healthier populations score higher on the PHCS compared to elderly who are managing chronic illness. This study examined the PHCS in three Midwestern samples: African American elders which revealed two separate factors in PHCS; Native Americans who evaluated the PHCS as a single construct; and a Spanish speaking sample. In the latter sample, findings suggest health competence may be a multi-dimensional construct. Further evaluation of the Spanish version of the PHCS and population characteristics are needed to measure health competence beliefs.

\section{Keywords}

Health Promotion, Self-Efficacy, Health Management, Latino/Hispanic Populations, Native Americans, African American Elders, Health Competence, Access to Health Services, Instrument Development, Pilot Study, Translation of Instruments

\section{Introduction}

Disparities in health because of inadequate access to services are intricately related to population characteristics of age, gender, ethnicity, and social capital [1]. Few measures of self-efficacy beliefs describe and explore cultural influences about competence beliefs of health behaviors and health outcomes [2] [3]. The Perceived Health Competence Scale (PHCS), developed by Smith, Wallston, and Smith [4] has not been explored with minority populations. Rather, the original validation of the PHCS was undertaken in middle class samples in Europe and the United States and recent use has not reported race and ethnicity in samples [5]-[7]. The psychometric evalu- 
ation of the PHCS in three different minority samples was undertaken to explore self-efficacy beliefs about general health management, health behaviors, and health outcomes; this evaluation utilized the PHSS-ACCESS instrument that was developed by the author in English and Spanish versions. A measure of self-efficacy beliefs about general health management has value to explore potential for enacting healthy behaviors and expectations about achieving desired health outcomes in minority populations at risk of health disparities.

\section{Cross-Cultural Exploration of the Perceived Health Competence Scale}

\subsection{Rationale}

Self-efficacy beliefs about general health management may be the best predictor of health outcomes especially in vulnerable minority populations [8]. Self-efficacy is an individual's belief of his/her ability to act and to accomplish a specific goal. It is important to evaluate self-efficacy to understand the potential for health behavior change and the achievement of health outcomes [9] [10]. Exploration of attitudes, behaviors, and influences in self-efficacy beliefs requires awareness of potential cultural bias in the psychometric properties of instruments when used with at-risk populations because of multi-faceted contributing factors surrounding health competence [11]. Further, it is important to understand potential communication barriers that may result in a person's feeling misunderstood [12]. For example, negatively worded items are difficult to interpret for the elderly, in translated instruments, and in ethnically diverse samples [11].

Aday [13] suggests vulnerable populations may lack opportunities and resources for self-management, are at risk of poor health because of their physical, social, or psychological conditions, lack social capital and support networks, and have competing demands in managing their health situations. The Institute of Medicine reports that minority populations experience more complex chronic illnesses, encounter language and cultural barriers, and are less likely to seek treatment for preventive care [14]. Specific examples in the management of acute myocardial infarction (AMI) comparing ethnically diverse minority populations to White counterparts indicate that elderly African Americans may wait longer for an electrocardiogram and are less likely to receive thrombolytic therapy. Native Americans are less likely to have angioplasty and bypass surgery and Mexican Americans receive fewer medications after AMI [14].

\subsection{Conceptual Framework}

The Behavioral Model for Vulnerable Populations (BMVP) guided this research [3]. The BMVP suggests that certain population characteristics predict and influence health behaviors and outcomes. These population characteristics are: 1) predisposing demographic predictor variables of age, gender, health beliefs, and social networks; 2) enabling predictor variables such as a regular source of care, transportation, and perceptions about barriers to access care; and 3) predictor variables of need such as the individual self-evaluated need for health services and self-evaluated general health status. The BMVP was tested with disadvantaged populations and facilitates the identification of social determinants of health outcomes for minority populations [3]. Few frameworks emphasize the unique cultural and environmental influences in the lives of minority populations' health competence beliefs [13].

\section{Purpose}

This study was undertaken to evaluate the psychometric properties of the PHCS in three different samples. Cross-cultural validation of the PHCS aims to explore the abstract concept of self-efficacy or perceptions about performing behaviors and achieving outcomes in general health management; different cultures may have different perceptions of control over health outcomes [15]. Self-efficacy, as measured with the PHCS, reflects capability of health management. In certain cultures, it is possible that management of health is not seen as a priority with other competing demands [1] [16]. The research question is: What are the psychometric properties of the PHCS in three ethnically diverse samples?

\section{Method}

This cross-sectional descriptive research was conducted in phases with participants from separate settings in the Midwest to explore the psychometric properties of the PHCS in three diverse samples. The three diverse sam- 
ples were 88 community dwelling African American elders, 131 Native Americans utilizing health services at a community health center, and a pilot study with 19 participants of a Spanish speaking sample attending a religious event. A triangulated approach to explore health competence beliefs in the Spanish speaking sample was conducted to pilot the Spanish version of the PHCS and explore individual health care experiences using an open-ended question and recording field notes [17] [18]. Institutional Review Board approval for each of the three data collection sites as individual studies was obtained from the University.

\subsection{Instrumentation}

\subsubsection{The Perceived Health Competence Scale}

The eight-item PHCS is a domain-specific measure of general health management self-efficacy beliefs scored with a Likert type scale. The self-efficacy beliefs combine both outcome and behavioral expectations as a single construct. In development of the scale, internal consistency coefficients ranged from 0.82 to 0.90 across samples. A higher PHCS score (possible range 8 - 40) suggests more health competence and higher self-efficacy beliefs about enacting behaviors to achieve desired health outcomes [4]. In validation of the concept of health competence, the PHCS reported those who are younger and healthier (not managing chronic disease) scored higher in their competency beliefs, correlated positively with other general measures of well-being, active coping styles, or mental adjustment, and showed promise predicting health behavior intentions.

The PHCS was a reliable predictor of future health intentions in a young sample of military women [19], in women with breast cancer [20], and college students [4]. Marks and Lutgendorf reported perceived health competence and personality factors predicted health behaviors in older (primarily Caucasian) adults [21]. Salyer, Flattery, Joyner, and Elswick reported positive relationships between the PHCS and quality of life in cardiac transplant recipients [22]. Lee and McCleary reported that the PHCS was reliable in assessing senior citizens' health practices [6]. Rueda and Perez-Garcia reported good PHCS scores and quality of life as the major predictors in management of hypertension, but race and ethnicity were not reported [7].

\subsubsection{The Perceived Health Status Scale-ACCESS}

The Perceived Health Status Scale-ACCESS (PHSS-ACCESS) was developed by the author to explore and validate the concept of health competence following the BMVP as suggested by Gelberg and colleagues [3]. The PHSS-ACCESS examines self-evaluated health status and perceived barriers in accessing health services as enabling population characteristics. It is scored with a five-point Likert type scale (response range 5 - 25) with higher scores indicating fewer perceived barriers to accessing health services. The PHSS-ACCESS examines self-evaluated health status as population characteristic indicative of the perceived need for health services as suggested with the BMVP. Respondents are asked to circle items from a list of five chronic health problems, indicate perceived general health status overall, and provide demographic information.

\subsubsection{Spanish Versions of the Instruments}

The two instruments (PHCS and PHSS-ACCESS) were translated to Spanish and piloted in this Midwestern setting. Translation of instruments is best accomplished with discussion and evaluation by community members to critique content, response choices, and to ensure cultural fit [23]. The Spanish versions were developed over time with forward and backward translation (A.S.G., personal communication, March 7, 2013). The team members for translation were from the local community and consisted of a Spanish professor of the sponsoring institution who also assisted with data collection, interpretation processes, and analysis. Other team members were Spanish speaking health care providers from the local public health services and a local priest serving as a liaison within the setting.

The PHCS Spanish version uses the five-point Likert type scale response and contains the same items as the English version. The first two items of the PHCS are negatively worded; with translation of the PHCS to Spanish, the ordering of items was changed to avoid negative wording at the beginning of the instrument.

The PHSS-ACCESS examines perceived barriers in accessing health services as enabling population characteristics as suggested with the BMVP. The Spanish version is scored with a three-point Likert type scale (response range 3 - 15), with higher scores suggesting no perceived barriers to accessing health services. The threepoint Likert type scale response was used for the Spanish speaking participants to decrease extreme response set bias [23]. Acculturation was estimated by number of years in the US, country of birth, and ethnicity [24]-[26]. 
The process of instrument development was explained in the training sessions for data collectors to confirm that interpretation was culturally congruent to examine health competence beliefs [8] [16].

\subsection{Samples}

Each setting utilized convenience sampling; adequate sample size was met for the African American elders and the Native Americans with 10 to 15 participants per item for the eight-item PHCS [18]. Selection within the African American elder sample was that those who were in attendance at the noon meal program were approached by the primary investigator and asked to complete the instrument. Selection within the Native American sample was that those who came to the Red Cliff Community Health Center for health services were asked to complete the instrument by a member of the tribe that was a student nurse of the sponsoring University and who also served on the Tribal Education Committee [27].

In all settings, the researcher and other data collectors offered to read the instrument to participants and participation was voluntary. Each setting used a small thank you gift appropriate to the setting. A gift card for local food vendors was used with the African American elder sample and a basket of fresh fruit was available for participants in the Native American sample during data collection. One-to-one data collection shows respect for participants and encourages participants to ask questions if an item is difficult to understand and share feelings about the instrument [23]. In establishing reliability and validity of instruments it is important to ask participants their feelings about the survey, the research process, and to obtain feedback about their personal experience [28].

With all samples, field notes were collected by the researcher and other data collectors; for example, they noted looks of puzzlement when reading items to participants and the number of times items were asked to be repeated. As the first two items of the PHCS are negatively worded, it was often necessary to repeat them, most often in the sample of African American elders.

Field notes for the pilot study of the 19 participants in the Spanish Speaking sample included an open-ended question asking respondents to share a "story" about past health care experiences. Selection criteria for the pilot study of the instruments in the Spanish Speaking sample was accomplished by having an announcement made during a program that Spanish speaking data collectors were available to speak with individuals who wished to complete a "survey" about their health care experiences. The potential participants then approached the Spanish speaking data collectors and volunteered to complete the "survey" with the data collector taking field notes. The Spanish speaking data collectors worked in teams of two so that the field notes could be written for future analysis.

\section{Results}

\subsection{Attributes of African American Elders Sample}

A convenience sample of 88 urban dwelling African American elders aged 65 to 96 years $(M=75.18, S D=6.72)$ attending an established noon meal program participated in this research; 78\% were female $(N=69)$. A description of the African American elder participants and scores are presented in Table 1. Participants had multiple chronic health problems $(M=2.11)$; $82 \%$ reported high blood pressure, 66\% reported arthritis, 30\% reported diabetes, $18 \%$ reported shortness of breath, and 16\% reported congestive heart failure. Sixty-eight percent agreed or strongly agreed with the statement "I believe my overall health is as good as it can be". The mean score for the five-item PHSS-ACCESS was 20.15 (SD = 3.33) indicating few problems with access to health services, an expected finding in this urban setting with public transportation readily available.

\subsection{Elderly African American PHCS Scores}

The PHCS Cronbach alpha was 0.65, which fell below the recommended acceptability to measure true individual differences; therefore an exploratory factor analysis was performed [17]. Two factors emerged with Eigenvalues greater than 1.00 explaining 61\% of the total cumulative variance. Component Loadings for PHCS Subscales are provided in Table 2 for the African American elders. Guided by the BMVP theoretical framework, the two components were named PHC-Behaviors and PHC-Outcomes, suggesting separate constructs about health competence as perception of control over ability to perform health behaviors and perception of control to achieve desired health outcomes as explained by Smith and colleagues in validation of the PHCS [4]. Negatively worded components clustered together as one component, similar to factor analysis and subscales reported by 
Table 1. Description of samples and scores.

\begin{tabular}{|c|c|c|c|c|c|c|}
\hline SAMPLE & $\begin{array}{c}\text { PHSS9 } \\
\text { HLTH-Status } \\
\% \text { Agreement or } \\
\text { Mean Score }(0-5)\end{array}$ & $\begin{array}{l}\text { Number of } \\
\text { Health Problems }\end{array}$ & $\begin{array}{l}\text { PHCS Total Score } \\
\text { Eight-item Scale }\end{array}$ & $\begin{array}{c}\text { ACCESS } \\
\text { Possible Range } \\
5-25\end{array}$ & $\begin{array}{l}\text { PHC-Behavior } \\
\text { Subscale } \\
\text { Score }\end{array}$ & $\begin{array}{l}\text { PHC-Outcomes } \\
\text { Subscale Score }\end{array}$ \\
\hline $\begin{array}{c}\text { Elderly } \\
\text { African American } \\
\text { (N88) }\end{array}$ & $\begin{array}{l}68 \% \\
\text { N60 } \\
\text { A/SA }\end{array}$ & M2.11 & $\begin{array}{l}\text { M28.24 } \\
\text { (SD 3.99) }\end{array}$ & $\begin{array}{l}\text { M20.15 } \\
\text { (SD 3.33) }\end{array}$ & $\begin{array}{l}\text { M15.95 } \\
(S D 2.20)\end{array}$ & $\begin{array}{l}\text { M12.38 } \\
\text { (SD 3.45) }\end{array}$ \\
\hline $\begin{array}{c}\text { Elderly } \\
\text { Native American } \\
\text { (n16) }\end{array}$ & $\begin{array}{c}75 \% \\
\text { N12 } \\
\text { m3.50 (SD 0.89) } \\
\text { R2-4 }\end{array}$ & $\begin{array}{c}\text { M2.37 } \\
(S D 1.13)\end{array}$ & $\begin{array}{c}\text { M28.31 } \\
\text { (SD 4.81) } \\
\text { R19-38 }\end{array}$ & $\begin{array}{l}\text { M20.06 } \\
\text { (SD 1.83) } \\
\text { R16-25 }\end{array}$ & $\begin{array}{c}\text { M15.31 } \\
\text { (SD 2.35) } \\
\text { R8-20 }\end{array}$ & $\begin{array}{l}\text { M13.00 } \\
\text { (SD 3.46) } \\
\text { R7-18 }\end{array}$ \\
\hline $\begin{array}{c}\text { TOTAL Native } \\
\text { American Sample } \\
\text { (N131) }\end{array}$ & $\begin{array}{l}45 \% \\
\mathrm{~A} / \mathrm{SA}\end{array}$ & $\begin{array}{c}\text { M1.17 } \\
\text { (SD 1.23) } \\
\text { R0-5 }\end{array}$ & $\begin{array}{c}\text { M28.22 } \\
\text { (SD 5.26) } \\
\text { R15-40 }\end{array}$ & $\begin{array}{c}\text { M20.30 } \\
\text { (SD 2.75) } \\
\text { R10-25 }\end{array}$ & $\begin{array}{c}\text { M14.90 } \\
\text { (SD 2.62) } \\
\text { R7-20 }\end{array}$ & $\begin{array}{c}\text { M13.31 } \\
\text { (SD 3.35) } \\
\text { R5-20 }\end{array}$ \\
\hline $\begin{array}{c}\text { Native American } \\
\text { Males } \\
\text { Age } \leq 64 \text { years } \\
\text { (n32) }\end{array}$ & $\begin{array}{c}\mathrm{m} 2.87 \\
\text { (SD 1.12) } \\
\text { R1-5 }\end{array}$ & $\begin{array}{c}\mathrm{m} 1.40 \\
(S D 1.41) \\
\text { R0-5 }\end{array}$ & $\begin{array}{c}\mathrm{m} 27.25 \\
(S D 4.78) \\
\mathrm{R} 17-37\end{array}$ & $\begin{array}{c}\mathrm{m} 19.31 \\
(S D 2.53) \\
\mathrm{R} 12-23\end{array}$ & $\begin{array}{c}\mathrm{m} 14.48 \\
(\mathrm{SD} 2.60) \\
\mathrm{R} 9-20\end{array}$ & $\begin{array}{c}\mathrm{m} 12.78 \\
(S D 2.80) \\
\mathrm{R} 7-18\end{array}$ \\
\hline $\begin{array}{c}\text { Native American } \\
\text { Females } \\
\text { Age } \leq 64 \text { years } \\
\text { (n83) }\end{array}$ & $\begin{array}{c}\mathrm{m} 3.08 \\
(S D 1.21) \\
\mathrm{R} 1-5\end{array}$ & $\begin{array}{c}\mathrm{m} 0.85 \\
(S D 0.98) \\
\mathrm{R} 0-3\end{array}$ & $\begin{array}{c}\mathrm{m} 28.57 \\
(S D 5.52) \\
\mathrm{R} 15-40\end{array}$ & $\begin{array}{c}\mathrm{m} 20.73 \\
(S D 2.88) \\
\mathrm{R} 10-15\end{array}$ & $\begin{array}{c}\mathrm{m} 15.00 \\
(S D 2.68) \\
\mathrm{R} 7-20\end{array}$ & $\begin{array}{c}\mathrm{m} 13.57 \\
(S D 3.52) \\
\mathrm{R} 5-20\end{array}$ \\
\hline
\end{tabular}

${ }^{*}$ Mean Age of 18 - 64 year old Native American Total Sample was $39.76(S D=13.62) ;{ }^{*} \mathrm{R}=$ Range of possible scores in scale reported when available, ${ }^{*} \mathrm{M}=$ total sample and ${ }^{*} \mathrm{~m}=$ subgroups within total sample.

Table 2. Component loadings for PHCS sub-scales.

\begin{tabular}{|c|c|c|c|}
\hline Item number & Item description/statement & \multicolumn{2}{|c|}{ Factor loading } \\
\hline & Component 1 PHC-Behaviors & AA elders & Native American \\
\hline PHCS3 & I handle myself well with respect to my health & 0.722 & 0.710 \\
\hline PHCS4 & $\begin{array}{l}\text { I am able to do things for my health } \\
\text { as well as most other people }\end{array}$ & 0.863 & 0.648 \\
\hline PHCS5 & $\begin{array}{l}\text { I succeed in the projects I undertake } \\
\text { to improve my health }\end{array}$ & 0.753 & 0.833 \\
\hline \multirow[t]{2}{*}{ PHCS8 } & $\begin{array}{l}\text { I'm generally able to accomplish my } \\
\text { goals with respect to my health }\end{array}$ & 0.699 & 0.774 \\
\hline & Component 2 PHC-Outcomes & & \\
\hline rPHCS1 & $\begin{array}{l}\text { It is difficult for me to find effective solutions } \\
\text { for my health problem that come my way }\end{array}$ & 0.870 & 0.815 \\
\hline rPHCS2 & $\begin{array}{l}\text { I find efforts to change things I don't } \\
\text { like about my health are ineffective }\end{array}$ & 0.640 & 0.839 \\
\hline rPHCS6 & $\begin{array}{l}\text { Typically, my plans for my health } \\
\text { don't work out well }\end{array}$ & 0.827 & $0.600^{*}$ \\
\hline rPHCS7 & $\begin{array}{l}\text { No matter how hard I try, my health } \\
\text { doesn't turn out the way I would like }\end{array}$ & 0.805 & $0.642^{*}$ \\
\hline
\end{tabular}

*NA sample rPHCS6 and rPHCS7 loaded on both components, higher in Component 2; rPHCS—denotes reverse scored items.

Bonetti and colleagues [29].

\subsubsection{PHCS-Subscale Factor 1: PHC-Behaviors Subscale}

Four items, all statements about health competence in behaviors, comprise the PHC-Behaviors Subscale. The four items are: Item 3, I handle myself well with respect to my health; Item 4, I am able to do things for my 
health as well as most other people; Item 5, I succeed in the project I undertake to improve my health; Item 8, I'm generally able to accomplish my goals with respect to my health. Reliability of this subscale was 0.754 ; scores ranged from 4.00 to 20.00 . The mean score for participants on this subscale was $15.95(S D=2.20)$.

\subsubsection{PHCS-Subscale Factor 2: PHC-Outcomes Subscale}

Four items, all reverse scored, are statements on expectations about achieving desired health outcomes in the PHC-Outcomes Subscale. They are: Item 1, It is difficult for me to find effective solutions for my health problems that come my way; Item 2, I find efforts to change things I don't like about my health are ineffective; Item 6, Typically, my plans for my health don’t work out well; Item 7, No matter how hard I try, my health doesn’t turn out the way I would like. Reliability of this subscale was 0.793. Scores ranged from 6.00 to 20.00. The mean score for participants on this subscale was $12.38(S D=3.45)$.

A moderate positive correlation was found with PHC-Behaviors and positive health status $(r(84)=0.27, p<$ 0.01) and the literature supports that those who feel good about their health status have higher scores in health competence beliefs [19] [20]. This sample demonstrated a moderately positive relationship with access to health services $(r(83)=0.51, p<0.01)$ as measured with the PHSS-ACCESS supporting the BMVP enabling characteristics of a regular source of care as predictive of health behavior change and achievement of desired health outcomes.

\subsection{Attributes of the Native American Sample}

A convenience sample of 131 Native Americans aged 18 to 87 who obtained health services at the Red Cliff Community Health Center participated in this phase of data collection. The Red Cliff Band of Lake Superior Chippewa are longstanding in the Native Sovereign Nation. Permission for entry into the setting was given through the Red Cliff Tribal Health Board Committee [27]. Participants had the PHCS and the PHSS-ACCESS instruments read to them by one of their own, a trusted member of the tribe who was on the Tribal Education Committee and a student nurse of the sponsoring University. Having a member of the community serve as data collector demonstrated respect for the participants and provided assistance with negatively worded items. A description of the Native American participants and scores are presented in Table 1.

The mean age of the sample was 43.69 years $(S D=16.71) ; 70.22 \%$ were female $(N=92)$. The sample was relatively healthy with only $1.17(S D=1.23)$ self-reported chronic health problem; 39\% reported no health problems. Seventy-two percent agreed or strongly agreed with the statement "I believe my overall health is as good as it can be" reflecting a relatively healthy sample as suggested on the website mission to promote health and wellness [27]. The PHSS-ACCESS five-item subscale mean score was $20.30(S D=2.75)$ indicating few problems with access to health services; this finding was to be expected in those living in membership with a Tribal Community with Health Services in a Native Sovereignty Tribal setting that promotes, plans, and provides for the health of members [27].

\subsection{Native American PHCS Scores}

The PHCS Cronbach alpha was 0.84 for this Native American sample and supports the PHCS as measuring a single construct of health competence. PHCS total mean scale score was $28.22(S D=5.26)$ suggesting that this Native American sample felt good about their health competence. To compare the subscale scores, an exploratory factor analysis was conducted. The PHC-Behaviors mean scale score was $14.90(S D=2.62)$ and the PHC-Outcomes mean scale score was $13.31(S D=3.35)$ without significant differences between the genders. These results are to be expected with a relatively young and healthy sample [4]. Component Loadings for PHCS Sub-scales are provided in Table 2 for the Native American sample.

\subsection{Attributes of Pilot Exploration with Spanish Speaking Sample}

The mean age of the 19 participants was $49.47(S D=23.83) ; 7$ were male and 12 were female. Of this Spanish speaking sample, 63\% described themselves as being from "Mexico". This pilot study sample of 19 participants had an average of less than one health problem, with 10 people reporting no chronic health problems. Ninetyfive percent of this sample agreed with the statement, "I believe my overall health is as good as it can be.” Thirty-two percent of the participants in this pilot study reported being overweight as a health concern. Perceptions 
of health may be difficult to measure in this Spanish speaking sample with a large proportion of women; for Latinas, the absence of chronic illness indicates good health and being overweight is a frequently reported health problem [15]. The PHSS-ACCESS subscale mean score was $12.36(S D=2.47)$ using a three-point Likert type scale (range 5 - 15) suggesting that this sample felt that they had good access to health services.

The focus of this pilot study was to explore the perceptions of health competence with the PHCS in a Spanish speaking sample to explore their self-efficacy beliefs. This was done by providing opportunities for the participants to talk with data collectors about the Spanish version of the PHCS and to share stories of their past experiences with health services, to describe their health status, and to describe perceived health competence with a specific situation. Data collectors, trained local community members, worked in teams and wrote field notes about the participant experiences. Immediately after the data collection, the data collectors met together with the primary investigator and the Spanish professor to share the stories they were told by the participants; field notes were interpreted as a group process to explore the findings. Three main themes were expressed during the analysis of the stories: 1) a lack of trust with the use of interpreters; 2) fear about misdiagnosis during health encounters because of language barriers; and 3) fear about future access to health services because of legal status, insurance, or consequences with employers finding out about health problems that may impair their working ability.

\subsection{Spanish Speaking PHCS Scores}

The PHCS Spanish version Cronbach alpha was 0.608; the small sample size limits further analysis. The means scale score for the eight-item scale was $28.00(S D=5.13$, variance 26.33). No conclusions can be drawn from this analysis; field notes were interpreted.

\section{Discussion}

The specific aim of this research was to explore the psychometric properties of the PHCS in three ethnically diverse samples to further investigate health competence beliefs. This study was guided by the BMVP and population characteristics that may predict health behaviors and outcomes; Bonetti and colleagues suggest that the measurement of beliefs about control over health is best examined with the theoretical framework guiding the study [29]. The BMVP guiding this study suggests competing needs, ability to navigate systems, community resources, and an individual's evaluated health status may influence health behaviors [3] more than age and health status as measured with the presence of chronic disease as suggested by past research [4] [5] [21] [30].

Validation of the abstract concept of health competence requires theoretical exploration of the elements of the construct within the population of interest to enhance understanding of barriers encountered in self-management. Few measurement tools exist to explore health competence beliefs in minority populations [2] and cultural tailoring is needed with interpretation of results to enhance understanding of self-management and potential for behavior change to achieve desired health outcomes. The PHCS shows promise for future research when population characteristics, cultural nuances, and health beliefs are explored.

\subsection{Reliability of the PHCS}

Reliability estimate calculation and the ability to observe true differences in the psychosocial construct of health competence are subject to random error in measurement related to characteristics within the respondents and the setting. Sources of random error affecting reliability in this cross-cultural exploration of the PHCS are the age of the respondents in the sample of African American elders, individual subjective perceptions of culture and past health experiences, the desire for respondents to provide a socially desirable response, and the small sample in the pilot study with the Spanish version of the PHCS [18]. The PHCS has four negatively worded items that may be difficult to interpret and respond to for elderly, socio-economically disadvantaged, and culturally diverse populations [11] [31] [32].

\subsubsection{African American PHCS Reliability}

In the sample of African American elders, the two factors that emerged suggest that this sample viewed the PHCS as two separate constructs; competency beliefs about behaviors and outcomes. Factor analysis is rarely reported for instruments that do not achieve the recommended reliability. Bonetti and colleagues reported the 
same two factor structure for the PHCS in samples of patients with chronic illness in Spain, Scotland, and Ireland [29].

\subsubsection{Native American PHCS Reliability}

In the Native American sample the eight-item PHCS proved to be a statistically reliable measure of health competence. These results are supported with past use of the PHCS in relatively healthy and younger populations [19] [20] [29] [33]. The use of a young, racially matched data collector and trusted member of the community may support the reliability and validity of these findings [14] [34].

\subsubsection{Spanish Speaking PHCS Reliability}

The PHCS did not achieve adequate reliability as a single construct scale. In a study conducted in Spain, Pastor and colleagues estimate reliability at 0.79 in a sample of 252 healthy people and 56 people with chronic disease [35]. The linguistics of the instrument in the Spanish sample and the instrument used in this study had certain variations. Validation of the construct of health competence is sensitive to cultural variation with population characteristics.

\subsection{Validation of the PHCS and Health Competence in Latino/Hispanic Populations}

Cultural nuances in measurement of variables suggest that within the Hispanic/Latino population self-management, coping with chronic diseases, and self-efficacy beliefs may not equate to Western European norms that suggest an individual has control over health outcomes [11]. Skaff and colleagues suggest that health competence is acquired over time and must be viewed in the "broader context of resources, priorities, social responsibilities, and level of autonomy” ([16], p. 308). This pilot sample was young and self-reported as healthy. Previous research has shown that Latinas believe that the presence of chronic disease does not determine health but is self-evaluated as "feeling well” and "being happy" [15]. Latinos believe in seeking care within the family rather than external health agencies, believe that spirits or "powerful others" are in control of health, are reluctant to complain about health problems [25] and that being able to work is more important than seeking health services and will work regardless of pain or illness (Personal Communication, S. V., June, 2012).

Diverse cultures perceive health in different ways and one-to-one interviews in this pilot study with the Spanish speaking sample were used to ask participants to share their health care experiences. The Spanish speaking sample shared that that language barriers were present in health care encounters and limited their understanding of the treatment plan needed to develop health competence and an individual self-management plan. Self-management skills to develop health competence require the development of a partnership with the provider; Holman and Lorig suggest communicating with providers and a regular source of care are needed for symptom management, especially in chronic health conditions [36]. The Spanish speaking sample in this study suggested that the language barriers impaired trust during their past experiences with a provider and that they were unsure about their diagnosis and plan for health care during the health care encounter. Language barriers impair listening to symptom description [37], heighten anxiety, and limit understanding between provider and those seeking care resulting in poor compliance and powerlessness to change behaviors to achieve health outcomes [12] [38].

The process of data collection matched Spanish speaking community members with respondents to share a story about their health. This method of research provided a trusting environment and provided rich data about an at risk population [34]. During debriefing, data collectors shared their own personal pride and satisfaction in facilitating the understanding of health needs in this community to begin to address health disparities. The data collectors share their enhanced awareness of their own enculturation and the acculturation processes of their community members [24].

\subsection{Limitations}

The convenience sampling procedures and small sample size limit the ability to achieve adequate reliability in measurement with the Spanish version of the PHCS and limit generalizations about the psychometric properties of the PHCS. The purpose of this study was cross-cultural exploration of psychometric properties of the PHCS to describe health competence in three diverse samples. Future research should evaluate the psychometric qualities of the Spanish version of the PHCS with a larger sample to explore health competence beliefs and self-efficacy beliefs about general health management. Future research should investigate the sensitivity of the PHCS 
to assess changes over time and after self-management interventions.

\subsection{Conclusion and Implications for Future Research}

Overall, the English version of the PHCS is a reliable instrument to measure self-efficacy beliefs about general health management and health competence beliefs. The pilot study of the Spanish version of the PHCS and interview data contribute to enhanced understanding of individual perceptions of health competence and self-efficacy beliefs in this Midwestern population. Comprehensive exploration of individual perceptions of health competence can be achieved with reliable measurement tools and focused attention to the population characteristics of ethnicity, race, culture, access to health services, and perceived health status or need for health care services. Future efforts to understand how individuals feel about their health competence and self-management beliefs may enhance communication about perceived barriers for health behaviors toward achieving health outcomes in minority and at-risk populations.

\section{Acknowledgements}

The author was a participant in the 2013 NLN Scholarly Writing Retreat sponsored by Pocket Nurse and the NLN Foundation for Nursing Education.

\section{References}

[1] Carlson, E. and Chamberlain, R. (2003) Social Capital, Health, and Health Disparities. Journal of Nursing Scholarship, 35, 325-331. http://dx.doi.org/10.1111/j.1547-5069.2003.00325.x

[2] Flaskerud, J., Lesser, J., Dixon, E., Anderson, N., Conde, F., Kim, S., et al. (2002) Health Disparities among Vulnerable Populations: Evolution of Knowledge over Five Decades in Nursing Research Publications. Nursing Research, 51, 74-85. http://dx.doi.org/10.1097/00006199-200203000-00003

[3] Gelberg, L., Andersen, R. and Leake, D. (2000) Healthcare Access and Utilization: The Behavioral Model for Vulnerable Populations: Application to Medical Care Use and Outcomes for Homeless People. Health Services Research, 34, 1273-1302.

[4] Smith, M., Wallston, K. and Smith, C. (1995) The Development and Validation of the Perceived Health Competence Scale. Health Education Research, 10, 51-64. http://dx.doi.org/10.1093/her/10.1.51

[5] Dempster, M. and Donnelly, M. (2008) Validity of the Perceived Health Competence Scale in a UK Primary Care Setting. Psychology, Health, and Medicine, 13, 123-127. http://dx.doi.org/10.1080/13548500701351984

[6] Lee, S. and McCleary, K. (2012) The Relationship between Perceived Health, Health Attitude, and Healthy Offerings for Seniors at Family Restaurants. Cornell Hospital Quarterly, XX, 1-12.

[7] Rueda, B. and Perez-Garcia, A. (2006) A Prospective Study of the Effects of Psychological Resources and Depression in Essential Hypertension. Journal of Health Psychology, 11, 129-140. http://dx.doi.org/10.1177/1359105306058868

[8] Lorig, K., Ritter, P. and Gonzalez, V. (2003) Hispanic Chronic Disease Self-Management: A Randomized CommunityBased Outcome Trial. Nursing Research, 52, 361-369. http://dx.doi.org/10.1097/00006199-200311000-00003

[9] Bandura, A. (1990) Conclusion: Reflections on Non-Ability Determinants of Competence. In Sternberg, R. and Kolligian, J., Eds., Competence Considered, Yale University Press, New Haven, 315-362.

[10] Zulkosky, K. (2009) Self-Efficacy: A Concept Analysis. Nursing Forum, 44, 93-102. http://dx.doi.org/10.1111/j.1744-6198.2009.00132.x

[11] Gonzalez-Calvo, J., Gonzalez, V.M. and Lorig, K. (1997) Cultural Diversity Issues in the Development of Valid and Reliable Measures of Health Status. Arthritis Care and Research, 10, 448-456. http://dx.doi.org/10.1002/art.1790100613

[12] Condon, B.B. (2008) Feeling Misunderstood: A Concept Analysis. Nursing Forum, 43, 177-190. http://dx.doi.org/10.1111/j.1744-6198.2008.00112.x

[13] Aday, L. (2001) At Risk in America: The Health and Health Care Needs of Vulnerable Populations in the United States. 2nd Edition, Jossey-Bass, San Francisco.

[14] Smedley, B., Stith, A. and Nelson, A. (2002) Unequal Treatment: Confronting Racial and Ethnic Disparities in Health Care. The National Academies Press, Washington DC.

[15] Hartweg, D.L. and Isabelli-Garcia, C. (2007) Health Perceptions of Low-Income, Immigrant Spanish-Speaking Latinas in the United States. Hispanic Health Care International, 5, 53-63. http://dx.doi.org/10.1891/154041507780978914 
[16] Skaff, M.M., Mullan, J.T., Fisher, L. and Chesla, C.A. (2003) A Contextual Model of Control Beliefs, Behavior, and Health: Latino and European Americans with Type 2 Diabetes. Psychology and Health, 18, 295-312. http://dx.doi.org/10.1080/0887044031000084049

[17] Burns, N. and Grove, S. (2009) The Practice of Nursing Research: Appraisal, Synthesis, and Generation of Evidence. 6th Edition, Saunders, St. Louis.

[18] Waltz, C., Strickland, O. and Lenz, E. (1991) Measurement in Nursing Research. 2nd Edition, F.A. Davis Company, Philadelphia.

[19] Agazio, J.G., Ephraim, P.M., Flaherty, N.B. and Gurney, C.A. (2002) Health Promotion in Active-Duty Military Women with Children. Women \& Health, 35, 65-82. http://dx.doi.org/10.1300/J013v35n01_05

[20] Arora, N.K., Johnson, P., Gustafson, D.H., McTavish, F., Hawkins, R.P. and Pingree, S. (2002) Barriers to Information Access, Perceived Health Competence, and Psychosocial Health Outcomes: Test of a Mediation Model in a Breast Cancer Sample. Patient Education and Counseling, 47, 37-46. http://dx.doi.org/10.1016/S0738-3991(01)00170-7

[21] Marks, G.R. and Lutgendorf, S.K. (1999) Perceived Health Competence and Personality Factors Differentially Predict Health Behaviors in Older Adults. Journal of Aging and Health, 11, 221-239. http://dx.doi.org/10.1177/089826439901100205

[22] Salyer, J., Flattery, M.P., Joyner, P.L. and Elswick, R.K. (2003) Lifestyle and Quality of Life in Long-Term Cardiac Transplant Recipients. The Journal of Heart and Lung Transplantation, 22, 309-321. http://dx.doi.org/10.1016/S1053-2498(02)00552-1

[23] Hendrickson, S.G. (2003) Beyond Translation...Cultural Fit. Western Journal of Nursing Research, 25, 593-608. http://dx.doi.org/10.1177/0193945903253001

[24] Buscemi, C. (2011) Acculturation: State of the Science in Nursing. Journal of Cultural Diversity, 18, 39-42.

[25] Purnell, L. (2013) Transcultural Health Care: A Culturally Competent Approach. 4th Edition, F. A. Davis, Philadelphia.

[26] Siatkowski, A.A. (2007) Hispanic Acculturation: A Concept Analysis. Journal of Transcultural Nursing, 18, 316-323. http://dx.doi.org/10.1177/1043659607305193

[27] Red Cliff Band of Lake Superior Chippewa (2006) Official Home Page. http://redcliff-nsn.gov/

[28] Shortridge-Baggett, L. (2001) Self-Efficacy: Measurement and Intervention in Nursing. Scholarly Inquiry for Nursing Practice: An International Journal, 15, 183-188.

[29] Bonetti, D., Johnston, M., Rodriguez-Marin, J., Pastor, M., Martin-Aragon, M., Doherty, E. and Sheehan, K. (2001) Dimensions of Perceived Control: A Factor Analysis of Three Measures and Examination of Their Relation to Activity Level and Mood in a Student and Cross-Cultural Patient Sample. Psychology and Health, 16, 655-674. http://dx.doi.org/10.1080/08870440108405865

[30] Boyer, A., Mira, M., Calatayud, N., Roig, S., Marin, J. and Bruehl, S. (2010) Control Beliefs, Coping, and Emotions: Exploring Relationships to Explain Fibromyalgia Health Outcomes. International Journal of Clinical and Health Psychology, 10, 450-476.

[31] Rasin, J. (1997) Measurement Issues with the Elderly. In: Frank-Stromborg, M. and Olsen, S., Eds., Instruments for Clinical Health-Care Research, 2nd Edition, Jones and Bartlett Publishers, Sudbury, 44-53.

[32] Weinrich, S., Boyd, M. and Powe, B. (1997) Tool Adaptation for Socioeconomically Disadvantaged Populations. In: Frank-Stromborg, M. and Olsen, S., Eds., Instruments for Clinical Health-Care Research, 2nd Edition, Jones and Bartlett Publishers, Sudbury, 20-28.

[33] Sinclair, V.G. (2001) Predictors of Pain Catastrophizing in Women with Rheumatoid Arthritis. Archives of Psychiatric Nursing, 15, 279-288. http://dx.doi.org/10.1053/apnu.2001.28686

[34] Flaskerud, J.H. and Nyamathi, A.M. (2000) Attaining Gender and Ethnic Diversity in Health Intervention Research: Cultural Responsiveness versus Resource Provision. Advances in Nursing Science, 22, 1-15. http://dx.doi.org/10.1097/00012272-200006000-00002

[35] Pastor, M., Martin-Aragon, M., Lopez-Roig, S., Terol, M., Pons, N., Lledo, A., et al. (2009) The Perceived Health Competence Scale: Psychometric Properties in a Spanish Sample. Ansiedad y Estres, 15, 85-96.

[36] Holman, H. and Lorig, K. (1992) Perceived Self-Efficacy in Self-Management of Chronic Disease. In: Schwartzer, R., Ed., Self-Efficacy: Thought Control of Action, Hemisphere Publishing, Washington DC, 305-323.

[37] Shipley, S.D. (2010) Listening: A Concept Analysis. Nursing Forum, 45, 125-134. http://dx.doi.org/10.1111/j.1744-6198.2010.00174.x

[38] Armstrong, T.S. (2003) Symptoms Experience: A Concept Analysis. Oncology Nursing Forum, 30, 601-606. http://dx.doi.org/10.1188/03.ONF.601-606 